\title{
On the oscillatory behaviour of solutions of nonlinear conformable fractional differential equations
}

\section{Hakan Adiguzel}

Department of Architecture and Urban Planning, Vocational School of Arifiye, Sakarya University of Applied Sciences, Arifiye 54580, Turkey

Received: 30 June 2019, Accepted: 15 October 2019

Published online: 30 October 2019.

\begin{abstract}
In this study, we consider the conformable fractional differential equations. Based on properties of the conformable derivative, we obtain some oscillation results for the equation. Finally, we present some examples to illustrate the main results.
\end{abstract}

Keywords: Fractional differential equations, conformable derivative, oscillation

\section{Introduction and Preliminaries}

Fractional differential equations are more general form of the classical differential equations. There are many applications in the applied science such as image processing, etc $[1,2]$. In the last few decades, a lot of attention was paid to finding the more suitable definitions of fractional derivatives. Some of them: Riemann- Liouville, Caputo, Riesz, Riesz-Caputo, Weyl, Grunwald- Letnikov, Hadamard, and Chen derivatives, etc. In 2014, R. Khalil et al. [3] have suggested a new fractional derivative, which is called the conformable derivative. Based on the conformable derivative, many authors investigated differential equations $[3,4,5,6,7]$. Very recently in [8], the authors considered linear conformable fractional differential equation and then they obtained some oscillation results. In fact, oscillation properties of solutions of the fractional(or integer) order differential(or difference) equations has been the subject of intensive investigation $[8,9,10,11,12,13,14,15$, $16,17,18,19,20,21,22]$. We are strongly motivated by [8] and literature and are concerned with the oscillatory behavior of solutions of the following conformable fractional differential equation:

$$
y^{(2 \alpha)}(t)+p(t) y^{(\alpha)}(t)+q(t) f(y(t))=0
$$

where $p \in C\left(\left[t_{0}, \infty\right), \mathbb{R}\right), q \in C\left(\left[t_{0}, \infty\right),[0, \infty)\right), 0<\alpha \leq 1$ and $f \in C(\mathbb{R}, \mathbb{R})$ with $u f(u)>0$ for $u \neq 0$ and there exists a constant $k>0$ such that $f(u) / u \geq k$ for all $u \neq 0$. As usual, a solution $y(t)$ of (1) is called oscillatory if the set of its zeros unbounded from above; otherwise, it is said to be nonoscillatory. Equation (1) is called oscillatory if all its solutions are oscillatory.

Definition 1. [8] The left conformable fractional derivative starting from $t_{0}$ of a function of $f:\left[t_{0}, \infty\right) \rightarrow \mathbb{R}$ of order $\alpha$ with $0<\alpha \leq 1$ is defined by

$$
\begin{aligned}
\left(T_{t_{0}}^{\alpha} f\right)(t) & =f^{(\alpha)}(t) \\
& =\lim _{\varepsilon \rightarrow 0} \frac{f\left(t+\varepsilon\left(t-t_{0}\right)^{1-\alpha}\right)-f(t)}{\varepsilon},
\end{aligned}
$$


when $\alpha=1$, this derivative of $f(t)$ coincides with $f^{\prime}(t)$. If $\left(T_{t_{0}}^{\alpha} f\right)(t)$ exists on $\left(t_{0}, t_{1}\right)$ then

$$
\left(T_{t_{0}}^{\alpha} f\right)\left(t_{0}\right)=\lim _{t \rightarrow t_{0}^{+}} f^{(\alpha)}(t)
$$

Definition 2. [8] Let $\alpha \in(0,1]$. Then the left conformable fractional integral of order $\alpha$ starting at $t_{0}$ is defined by

$$
\left(I_{t_{0}}^{\alpha} f\right)(t)=\int_{t_{0}}^{t}\left(s-t_{0}\right)^{\alpha-1} f(s) d s=\int_{t_{0}}^{t} f(s) d_{t_{0}}^{\alpha} s
$$

If the conformable fractional integral of a given function $f$ exists, we call that $f$ is $\alpha$ - integrable.

Lemma 1. [4] If $\alpha \in(0,1]$ and $f \in C^{1}\left(\left[t_{0}, \infty\right), \mathbb{R}\right)$, then for all $t>t_{0}$, we have

$$
I_{t_{0}}^{\alpha} T_{t_{0}}^{\alpha}(f)(t)=f(t)-f\left(t_{0}\right)
$$

and

$$
T_{t_{0}}^{\alpha} I_{t_{0}}^{\alpha}(f)(t)=f(t)
$$

\section{Lemma 2.[3]}

(1) $T_{t_{0}}^{\alpha}(a f+b g)=a T_{t_{0}}^{\alpha}(f)+b T_{t_{0}}^{\alpha}(g)$ for all real constant $a, b$.

(2) $T_{t_{0}}^{\alpha}(f g)=f T_{t_{0}}^{\alpha}(g)+g T_{t_{0}}^{\alpha}(f)$.

(3) $T_{t_{0}}^{\alpha}\left(t^{p}\right)=p t^{p-\alpha}$, for all $p$.

(4) $T_{t_{0}}^{\alpha}(f / g)=\left(g T_{t_{0}}^{\alpha}(f)-f T_{t_{0}}^{\alpha}(g)\right) / g^{2}$.

(5) $T_{t_{0}}^{\alpha}(c)=0$, where $c$ is a constant.

Lemma 3. [5] Let $f, g:\left[t_{0}, t_{1}\right] \rightarrow \mathbb{R}$ be two functions such that $f g$ is differentiable. Then

$$
\int_{t_{0}}^{t_{1}} f(s) g^{(\alpha)}(s) d_{t_{0}}^{\alpha} s=\left.f(s) g(s)\right|_{t_{0}} ^{t_{1}}-\int_{t_{0}}^{t_{1}} g(s) f^{(\alpha)}(s) d_{t_{0}}^{\alpha} s .
$$

In this paper, we introduce a class of functions $Y$. We say that a function $\Phi(t, s, l)$ belongs to the function class $Y$ denoted by $\Phi \in Y$, if $\Phi \in C(E, \mathbb{R})$, where $E=\left\{(t, s, l): t_{0} \leq l \leq s \leq t<\infty\right\}$ which satisfies $\Phi(t, t, l)=0, \Phi(t, l, l)=0$, $\Phi(t, s, l) \neq 0$ for $l<s<t$, and has the partial derivative $\partial^{\alpha} \Phi / \partial s^{\alpha}$ on $E$ such that $\partial^{\alpha} \Phi / \partial s^{\alpha}$ is locally $\alpha-$ integrable with respect to $s$ in $E$ and satisfies

$$
\frac{\partial^{\alpha} \Phi(t, s, l)}{\partial s^{\alpha}}=\phi(t, s, l) \Phi(t, s, l)
$$

Next, we define the operator

$$
A(g ; l, t)=\int_{l}^{t} \Phi^{2}(t, s, l) g(s) d_{t_{0}}^{\alpha} s \text { for } t \geq s \geq l \geq t_{0} \text { and } g \in C([0, \infty), \mathbb{R}) .
$$

It is easy to verify that linear operator $A(. ; l, t)$ satisfies

$$
A\left(g^{(\alpha)} ; l, t\right)=-2 A(\phi g ; l, t) \text { for } g \in C^{\alpha}([0, \infty), \mathbb{R}) .
$$

\section{Main Results}

In this section, we present some oscillation criteria for the equation (1). 
Theorem 1. If $p(t)<0$ on $\left[t_{0}, \infty\right)$ is such that

$$
\lim _{t \rightarrow \infty}\left[\frac{1}{4} \int_{T}^{t}\left(4 k q(s)-p^{2}(s)\right) d_{t_{0}}^{\alpha} s\right]=\infty
$$

where $T \geq t_{0}$. Then any solution of $(1)$ is oscillatory.

Proof. On the contrary, suppose that Equation (1) has a nonoscillatory solution $y(t)$. Then without loss of generality, we may assume that $y(t)$ is a eventually positive solution of (1). Define the following Riccati function for $t \geq t_{0}$

$$
\omega(t)=-\frac{y^{(\alpha)}(t)}{y(t)} .
$$

Using the properties of conformable fractional derivative, we have

$$
\begin{aligned}
\omega^{(\alpha)}(t) & =-\frac{y^{(2 \alpha)}(t) y(t)-\left(y^{(\alpha)}(t)\right)^{2}}{y^{2}(t)} \\
& =\frac{p(t) y^{(\alpha)}(t)+q(t) f(y(t))}{y(t)}+\frac{\left(y^{(\alpha)}(t)\right)^{2}}{y^{2}(t)} \\
& \geq-p(t) \omega(t)+k q(t)+\omega^{2}(t) .
\end{aligned}
$$

Hence for every $t, T$ with $t \geq T \geq t_{0}$, we have

$$
\begin{aligned}
\omega(t) & \geq \omega(T)+\int_{T}^{t}\left(\omega^{2}(s)-p(s) \omega(s)+k q(s)\right) d_{t_{0}}^{\alpha} s \\
& =\omega(T)+\int_{T}^{t}\left(\omega(s)-\frac{p(s)}{2}\right)^{2} d_{t_{0}}^{\alpha} s+\frac{1}{4} \int_{T}^{t}\left(4 k q(s)-p^{2}(s)\right) d_{t_{0}}^{\alpha} s
\end{aligned}
$$

From (5), we get for $t \geq t_{1} \geq T$

$$
\omega(t) \geq \int_{t_{1}}^{t}\left(\omega(s)-\frac{p(s)}{2}\right)^{2} d_{t_{0}}^{\alpha} s
$$

Define

$$
H(t)=\int_{t_{1}}^{t}\left(\omega(s)-\frac{p(s)}{2}\right)^{2} d_{t_{0}}^{\alpha} s
$$

then we have $\omega(t)>H(t)>0$ on $\left[t_{1}, \infty\right)$. Hence

$$
H^{(\alpha)}(t)=\left(\omega(t)-\frac{p(t)}{2}\right)^{2}>\omega^{2}(t)>H^{2}(t)
$$

since $p(t)<0$. Thus,

$$
1<\frac{H^{(\alpha)}(t)}{H^{2}(t)}
$$

and we obtain

$$
\int_{t_{1}}^{t} d_{t_{0}}^{\alpha} s<\frac{1}{H\left(t_{1}\right)}-\frac{1}{H(t)}<\frac{1}{H\left(t_{1}\right)} .
$$

Letting $t \rightarrow \infty$ in (9), we conclude that

$$
\lim _{t \rightarrow \infty} \int_{t_{1}}^{t} d_{t_{0}}^{\alpha} s<\frac{1}{H\left(t_{1}\right)}
$$


This is a contradiction. Then the proof is complete.

Theorem 2. If $p(t)<0$ on $\left[t_{0}, \infty\right)$ and there exists a positive function $g \in C^{\alpha}\left[t_{0}, \infty\right)$ such that

$$
\lim _{t \rightarrow \infty} \int_{t_{0}}^{t} \frac{1}{g(s)} d_{t_{0}}^{\alpha} s=\infty
$$

and

$$
\lim _{t \rightarrow \infty}\left(-\frac{1}{4} \int_{T}^{t}\left(p^{2}(s) g(s)+\frac{\left(g^{(\alpha)}(s)\right)^{2}}{g(s)}-2 p(s) g^{(\alpha)}(s)-4 k g(s) q(s)\right) d_{t_{0}}^{\alpha} s+\frac{g^{(\alpha)}(t)}{2}\right)=\infty
$$

for every $T \geq t_{0}$, then any solution of (1) is oscillatory.

Proof. On the contrary, suppose that Equation (1) has a nonoscillatory solution $y(t)$. Then without loss of generality, we may assume that $y(t)$ is a eventually positive solution of (1). Define the following Riccati function for $t \geq t_{0}$

$$
\omega(t)=-g(t) \frac{y^{(\alpha)}(t)}{y(t)}
$$

Then we get

$$
\begin{aligned}
\omega^{(\alpha)}(t) & =-g^{(\alpha)}(t) \frac{y^{(\alpha)}(t)}{y(t)}-g(t)\left[\frac{y^{(2 \alpha)}(t) y(t)-\left(y^{(\alpha)}(t)\right)^{2}}{y^{2}(t)}\right] \\
& =\frac{g^{(\alpha)}(t)}{g(t)} \omega(t)-g(t)\left[\frac{y^{(2 \alpha)}(t)}{y(t)}-\frac{\left(y^{(\alpha)}(t)\right)^{2}}{y^{2}(t)}\right] \\
& =\frac{g^{(\alpha)}(t)}{g(t)} \omega(t)+g(t) p(t) \frac{y^{(\alpha)}(t)}{y(t)}+g(t) q(t) \frac{f(y(t))}{y(t)}+g(t) \frac{\left(y^{(\alpha)}(t)\right)^{2}}{y^{2}(t)} \\
& \geq \frac{1}{g(t)}\left(g^{(\alpha)}(t) \omega(t)-g(t) p(t) \omega(t)+\omega^{2}(t)\right)+k g(t) q(t)
\end{aligned}
$$

Now for $\left[t_{0}, \infty\right)$, defining

$$
H(t)=\omega(t)+\frac{1}{2} g^{(\alpha)}(t)
$$

we have

$$
\omega^{(\alpha)}(t) \geq \frac{1}{g(t)}\left(\left(H(t)-\frac{p(t) g(t)}{2}\right)^{2}-\left(\frac{p(t) g(t)}{2}\right)^{2}-\frac{\left(g^{(\alpha)}(t)\right)^{2}}{4}+\frac{p(t) g(t) g^{(\alpha)}(t)}{2}\right)+k g(t) q(t)
$$

that is

$$
\omega^{(\alpha)}(t) \geq \frac{1}{g(t)}\left(H(t)-\frac{p(t) g(t)}{2}\right)^{2}-\frac{p^{2}(t) g(t)}{4}-\frac{\left(g^{(\alpha)}(t)\right)^{2}}{4 g(t)}+\frac{p(t) g^{(\alpha)}(t)}{2}+k g(t) q(t)
$$


For every $t \geq T \geq t_{0}$, we get

$$
\begin{aligned}
\omega(t) & \geq \omega(T)+\int_{T}^{t} \frac{1}{g(s)}\left(H(s)-\frac{p(s) g(s)}{2}\right)^{2} d_{t_{0}}^{\alpha} s \\
& -\frac{1}{4} \int_{T}^{t}\left(p^{2}(s) g(s)+\frac{\left(g^{(\alpha)}(s)\right)^{2}}{g(s)}-2 p(s) g^{(\alpha)}(s)-4 k g(s) q(s)\right) d_{t_{0}}^{\alpha} s
\end{aligned}
$$

Using (13) in (14),

$$
\begin{aligned}
H(t) & \geq \omega(T)+\int_{T}^{t} \frac{1}{g(s)}\left(H(s)-\frac{p(s) g(s)}{2}\right)^{2} d_{t_{0}}^{\alpha} s \\
& -\frac{1}{4} \int_{T}^{t}\left(p^{2}(s) g(s)+\frac{\left(g^{(\alpha)}(s)\right)^{2}}{g(s)}-2 p(s) g^{(\alpha)}(s)-4 k g(s) q(s)\right) d_{t_{0}}^{\alpha} s \\
& +\frac{g^{(\alpha)}(t)}{2}
\end{aligned}
$$

From (11), we have for $t \geq t_{1}>T$

$$
H(t)>\int_{t_{1}}^{t} \frac{1}{g(s)}\left(H(s)-\frac{p(s) g(s)}{2}\right)^{2} d_{t_{0}}^{\alpha} s
$$

Now define a function $Q$ by

$$
Q(t)=\int_{t_{1}}^{t} \frac{1}{g(s)}\left(H(s)-\frac{p(s) g(s)}{2}\right)^{2} d_{t_{0}}^{\alpha} s .
$$

Using $p(t)<0$, we get $H(t)>Q(t)>0$. Hence,

$$
\begin{aligned}
Q^{(\alpha)}(t) & =\frac{1}{g(t)}\left(H(t)-\frac{p(t) g(t)}{2}\right)^{2} \\
& \geq \frac{1}{g(t)}\left(Q(t)-\frac{p(t) g(t)}{2}\right)^{2} \\
& >\frac{1}{g(t)} Q^{2}(t)
\end{aligned}
$$

That is

$$
\frac{1}{g(t)}<\frac{Q^{(\alpha)}(t)}{Q^{2}(t)}
$$

From (15), we have

$$
\int_{T_{1}}^{t} \frac{1}{g(s)} d s<\frac{1}{Q\left(T_{1}\right)}-\frac{1}{Q(t)}<\frac{1}{Q\left(T_{1}\right)} .
$$

This is a contradiction. So the proof is complete.

Theorem 3. If there exists a $\Phi \in Y$ such that the inequality

$$
\limsup _{t \rightarrow \infty} A\left(k q-\left(\phi-\frac{p}{2}\right)^{2} ; t_{1}, t\right)>0
$$

holds, where $\phi=\phi(t, s, l)$ and A are defined by (2) and (3), respectively. Then Equation (1) is oscillatory. 
Proof. On the contrary, suppose that Equation (1) has a nonoscillatory solution $y(t)$. Then without loss of generality, we may assume that $y(t)$ is a eventually positive solution of (1). Define the following Riccati function for $t \geq t_{0}$

$$
\omega(t)=\frac{y^{(\alpha)}(t)}{y(t)} .
$$

Then we get

$$
\begin{aligned}
\omega^{(\alpha)}(t) & =\frac{y^{(2 \alpha)}(t) y(t)-\left(y^{(\alpha)}(t)\right)^{2}}{y^{2}(t)} \\
& =\frac{-p(t) y^{(\alpha)}(t)-q(t) f(y(t))}{y(t)}-\frac{\left(y^{(\alpha)}(t)\right)^{2}}{y^{2}(t)} \\
& \leq-p(t) \omega(t)-k q(t)-\omega^{2}(t) .
\end{aligned}
$$

That is

$$
k q(t) \leq-\omega^{(\alpha)}(t)-p(t) \omega(t)-\omega^{2}(t)
$$

Applying $A\left(. ; t_{1}, t\right)\left(t>t_{1} \geq t_{0}\right)$ to (19), we get

$$
A\left(k q ; t_{1}, t\right) \leq-A\left(\omega^{(\alpha)} ; t_{1}, t\right)-A\left(p \omega ; t_{1}, t\right)-A\left(\omega^{2} ; t_{1}, t\right) .
$$

Then we have

$$
A\left(k q ; t_{1}, t\right) \leq 2 A\left(\phi \omega ; t_{1}, t\right)-A\left(p \omega ; t_{1}, t\right)-A\left(\omega^{2} ; t_{1}, t\right) .
$$

and

$$
\begin{aligned}
A\left(k q ; t_{1}, t\right) & \leq A\left(\left(\phi-\frac{p}{2}\right)^{2}-\left(\omega-\left(\phi-\frac{p}{2}\right)\right)^{2} ; t_{1}, t\right) \\
& \leq A\left(\left(\phi-\frac{p}{2}\right)^{2} ; t_{1}, t\right)
\end{aligned}
$$

Hence

$$
A\left(k q-\left(\phi-\frac{p}{2}\right)^{2} ; t_{1}, t\right) \leq 0
$$

for $t>t_{1} \geq t_{0}$, which contradicts (16). Therefore, all solutions of Equation (1) are oscillatory. So the proof is complete.

\section{Applications}

In this section, we give some example for the illustrate the main results.

Example 1. Consider the following fractional differential equation

$$
y^{(2 \alpha)}-\frac{1}{t} y^{(\alpha)}(t)+e^{t} y(t)=0, t>0
$$


with $\alpha=0,7$. The equation (20) corresponds with (1), $p(t)=-1 / t ; q(t)=e^{t}$ and $f(t)=t$. Then we have $p(t)<0$; $q(t)>0$ and $f(t) / t=1=k$. Hence for every $T \geq t_{0}=1$

$$
\begin{aligned}
\lim _{t \rightarrow \infty}\left[\frac{1}{4} \int_{T}^{t}\left(4 k q(s)-p^{2}(s)\right) d_{t_{0}}^{\alpha} s\right] & =\lim _{t \rightarrow \infty}\left[\frac{1}{4} \int_{T}^{t}(s-1)^{\alpha-1}\left(4 e^{s}-\frac{1}{s^{2}}\right) d s\right] \\
& =\infty
\end{aligned}
$$

Then every solution of the equation (20) is oscillatory by Theorem 1.

Example 2. Consider the following equation

$$
y^{(2 \alpha)}-\frac{2}{t} y^{(\alpha)}(t)+\left(1+\frac{2}{t^{2}}\right) y(t)=0, t>0 .
$$

with $\alpha=1$. Then we have $p(t)=-2 / t ; q(t)=1+2 / t^{2}$ and $f(t)=t$. Hence we get

$$
\begin{aligned}
\lim _{t \rightarrow \infty}\left[\frac{1}{4} \int_{T}^{t}\left(4 k q(s)-p^{2}(s)\right) d_{t_{0}}^{\alpha} s\right] & =\lim _{t \rightarrow \infty}\left[\frac{1}{4} \int_{T}^{t}(s-1)^{\alpha-1}\left(4\left(1+2 / t^{2}\right)-(-2 / t)^{2}\right) d s\right] \\
& =\lim _{t \rightarrow \infty}\left[\int_{T}^{t}\left(\left(1+2 / t^{2}\right)-\frac{(-2 / t)^{2}}{4}\right) d s\right] \\
& =\infty .
\end{aligned}
$$

Then every solution of the equation (21) is oscillatory by Theorem 1.

Remark. [8] The singularity of integral at $t_{0}$ has no affection on the oscillation of conformable fractional differential equations since oscillation is a qualitative property at infinite. The results are still true if the lower bound of the any integration is replaced by any other point larger than $t_{0}$.

Remark. As in the example [10], we have the equation (21). One can see that our results are original complementing and generalizing on already existing results in the literature of integer order equations.

\section{Competing interests}

The authors declare that they have no competing interests.

\section{Authors' contributions}

All authors have contributed to all parts of the article. All authors read and approved the final manuscript.

\section{References}

[1] Diethelm K., The Analysis of Fractional Differential, Springer, Berlin, (2010).

[2] Podlubny I., Fractional Differential Equations, Press, San Diego, (1999).

[3] Khalil, R., Al Horani, M., Yousef, A., \& Sababheh, M. A new definition of fractional derivative. Journal of Computational and Applied Mathematics, 264, pp. 65-70, (2014).

[4] Abdeljawad, T. On conformable fractional calculus, Journal of Computational and Applied Mathematics, 279, pp. 57-66, (2015). 
[5] Lazo, M. J., Torres, D. F., Variational calculus with conformable fractional derivatives, IEEE/CAA Journal of Automatica Sinica, 4(2), pp. 340-352, (2017).

[6] Atangana, A., Baleanu, D., and Alsaedi, A., New properties of conformable derivative, Open Mathematics, 13(1), pp. 889-898, (2015).

[7] Zhao, D., and Luo, M., General conformable fractional derivative and its physical interpretation, Calcolo, 54(3), pp. 903-917, (2017).

[8] Shao, J., \& Zheng, Z. Kamenev Type Oscillatory Criteria for Linear Conformable Fractional Differential Equations. Discrete Dynamics in Nature and Society, 2019. (2019).

[9] Mısır, A., \& Öğrekçi, S. Oscillation Theorems for Second-Order Nonlinear Differential Equations. Gazi University Journal of Science, 29(4), pp. 929-935. (2016).

[10] Abdullah, H. K. A note on the oscillation of second order differential equations. Czechoslovak Mathematical Journal, 54(4), pp. 949-954.(2004).

[11] Tunc, E. \& Tunc, O. On the oscillation of a class of damped fractional differential equations. Miskolc Mathematical Notes, 17(1), pp. 647-656. (2016).

[12] Muthulakshmi, V., \& Pavithra, S. Interval oscillation criteria for forced fractional differential equations with mixed nonlinearities. Glob. J. Pure Appl. Math., 13(9), pp. 6343-6353. (2017).

[13] Alzabut, J., Abdeljawad, T., \& Alrabaiah, H. Oscillation criteria for forced and damped nabla fractional difference equations. Journal of Computational Analysis and Applications, 24(8), pp. 1387-1394. (2018).

[14] Abdalla, B. Oscillation of differential equations in the frame of nonlocal fractional derivatives generated by conformable derivatives. Advances in Difference Equations, 2018(1), 107. (2018).

[15] Abdalla, B., Alzabut, J., \& Abdeljawad, T. On the oscillation of higher order fractional difference equations with mixed nonlinearities. Hacettepe Journal of Mathematics and Statistics, 47(2), pp. 207-217. (2018).

[16] Kısalar, S., Yıldı, M. K., \& Aktoprak, E. Oscillation of higher order fractional nonlinear difference equations. International Journal of Difference Equations, 10(2), pp. 201-212. (2015).

[17] Sun, Y.G. New Kamenev-type oscillation criteria for second-order nonlinear differential equations with damping. Journal of Mathematical Analysis and Applications. 291, pp. 341-351 (2004).

[18] Jiang, C., Tian, Y., Jiang, Y., \& Li, T. Some oscillation results for nonlinear second-order differential equations with damping. Advances in Difference Equations, 2015(1), 354. (2015).

[19] Ögrekçi, S., Misir, A., \& Tiryaki, A. On the oscillation of a second-order nonlinear differential equations with damping. Miskolc Mathematical Notes, 18(1), pp. 365-378. (2017).

[20] Huang, Y., \& Meng, F. Oscillation of second-order nonlinear ODE with damping. Applied mathematics and computation, 199(2), pp. 644-652. (2008).

[21] Y. Bolat, On the oscillation of fractional-order delay diferential equations with constant coefcients, Communications in Nonlinear Science and Numerical Simulation, 19(11), pp. 3988-3993, (2014).

[22] D.-X. Chen, Oscillation criteria of fractional diferential equations, Advances in Difference Equations, vol. 2012, article 33, 18 pages, (2012). 